\author{
Renu B Pattanshetty and Ceona \\ Chopde $^{*}$ \\ Department of cardio-pulmonary physiotherapy, \\ Kleu Institute of Physiotherapy, Medical College \\ Campus, Belagavi-590010, Karnataka, India
}

Dates: Received: 28 June, 2016; Accepted: 25 July, 2016; Published: 26 July, 2016

*Corresponding author: Dr. Ceona Chopde, Post Graduate Student, Department of Cardio-Pulmonary Physiotherapy, Kleu Institute of Physiotherapy. Jn Medical College Campus, Belagavi-590010, Karnataka, India, Tel: 91-7353789300; E mail: ceonaphysio.7@gmail.com

www.peertechz.com

ISSN: 2455-5487

Keywords: Cancer-related fatigue; Physical activity; Anxiety; Depression; Quality of life; Pilates; Taichi, acupuncture

\author{
Review Article
}

\section{Role of Physiotherapy in Cancer- Related Fatigue in Cancer Survivors - A Narrative Review}

\section{Introduction}

Fatigue is one of the most common problems experienced by cancer patients [1]. Fatigue is a normal and necessary instrument of physiologic self-regulation and protects the body from exaggerated or harmful efforts. It becomes pathologic when it appears during normal activities and does not improve after rest [2]. It is one of the most prevalent symptoms of cancer patients receiving radiation therapy, cytotoxic chemotherapy or biological response modifiers [3-6]. It was reported that $75 \%$ of patients with various solid tumors (in which 48 of 95 had metastatic disease) had significantly increased fatigue score when compared with control population [7]. Also, individuals who survive cancer, report fatigue as a problem, months to years after treatment ends [8]. Hence, cancer-related fatigue is defined as a persistent, subjective sense of tiredness related to cancer or cancer treatment that interferes with usual functioning $[9,10]$, can be in terms of perceived energy, mental capacity and psychological status $[11,12]$.

The specific causes that are involved in the development of cancer-related fatigue are not completely known. However, physiological factors that may cause fatigue include anaemia, cancer therapy, cachexia, tumour burden and the release of cytokines. Psychological factors like anxiety, depression, difficulty sleeping has also shown to play an important role and low degrees of physical functioning [13]. Fatigue is inversely associated with activity level and functional capacity. Physical fatigue also results from alterations in the aerobic and anaerobic pathways caused by cancer treatment. In aerobic pathways, several functional and anatomic changes occur due to cancer treatment and hence affect the oxygen supply to the cells. Alteration of the bronchial tree, lung and plasma volume, pulmonary perfusion, alveolar surface, heart function, red blood cell (RBC) count and concentration of oxidative enzyme in muscle cells, reduces oxygen transport. Chemotherapy can damage bone marrow and impairs the production of RBCs. The anaemia caused by such treatments decrease the oxygen supply to the cells. Cardiotoxic cytostatic agents may cause a reduction of cardiac output and result in an impairment of blood supply to the muscles. Lung volume loss caused by disease or/and as a sequel of treatment may alter ventilation-perfusion ratio causing impairment in blood oxygenation. All these factors contribute to reduction in oxygen delivery to the cells and limit the availability of oxygen for utilization in the synthesis of ATP at the mitochondrial crests. Therefore, cells obtain energy through anaerobic glycolysis producing less ATP with lactic acid as its end product. Lactic acid accumulation in the cells decreases intracellular and interstitial $\mathrm{pH}$ leading to additional metabolic stress. The combined burden of long lasting increase in heart rate and respiratory work, less effective energy production and metabolic acidosis can lead to tiredness, reduced stamina and inability to carry out intense physical effort [14].

It has been shown that sedentary patients with breast cancer, have reduced exercise tolerance and report higher degrees of fatigue during cancer treatment than more physically fit patients [15]. However, a consistent decrease in the amount of daily activity over the lengthy period of cancer treatment may lead to a reduced tolerance for normal activity and may develop high levels of fatigue [16].

Hence, the aim of this study was to explore the role of physiotherapy among cancer survivors experiencing cancerrelated fatigue. Literature searches were performed using electronic databases including Medline, Cochrane, Highwire Press and Pubmed using key words "cancer-related fatigue", "physical activity", "aerobic training", "taichi", "cancer survivors" and "resistance training".

\section{Screening and assessment for cancer-related fati- gue}

Recent advances in cancer screening and treatment have resulted in an expanding number of cancer survivors. A majority of patients experience some levels of fatigue during their course of treatment; however, approximately $30 \%$ of patients endure persistent fatigue for a number of years after treatment $[17,18]$. Patients with cancer can easily and reliably rate their fatigue and its severity on a simple 
numeric scale ranging from 0 -10 (0-no fatigue; 10 -worst fatigue imaginable), mild fatigue is indicated as a score of 1-3, moderate fatigue as 4-6 and severe fatigue as 7-10 [19,20]. Patients who report moderate to severe fatigue should undergo a comprehensive and focused assessment. There are unidimensional and multidimensional tools to assess/ measure cancer-related fatigue.

The unidimensional tools for screening include, Symptom Distress Scale devised by McCorkle and Quint-Benoliel which is a single-item measure of fatigue and is simple to administer [21]. Rotterdam Symptom Checklist devised by de Haes et al., has shown to measure distress. It is a single-item measure which is well validated for cancer patients [22]. Another tool to measure quality of life (QOL) is European Organization for Research and Treatment of Cancer Quality of Life Questionnaire (EORTC QOL-C30) which is a 30item QOL questionnaire with 3 items related to fatigue and measures symptom intensity during past week with internal consistency of $\alpha=0.54-0.86$ and is validated in cancer population [23]. Medical Outcomes Study 36-item short form health survey devised by Mc Horney et al., is a 4 -item subscale that measures fatigue frequency in past 4 weeks. It is useful for measuring general health status though not specifically designed to measure cancer-related fatigue [24]. MD Anderson Symptom Inventory devised by Cleeland et al., is a single item fatigue measure from 25 -item scale that measures fatigue severity in previous 24 hours with internal consistency of $\alpha=0.85$ 0.87 and is specifically designed to measure cancer related fatigue [25]. Zung Self-Rating Depression Scale measures fatigue frequency (none/little of the time to most/all of the time) during the preceding week devised by Kirsh et al., which contains a single fatigue item assessed on a 4-point Likert scale and cannot measure cancer related fatigue severity [26]. Rhoten Fatigue Scale is another scale which is simple and easy to use but is limited to severity assessment and has not shown to measure cancer related fatigue. It contains $0-10$ point graphic rating scale and measures fatigue severity [26].

Multi-item unidimensional measures of cancer related fatigue include, Fatigue Scale of the Profile of Mood States which measures presence and intensity of fatigue during the past week on a 5-point scale with 8 -item and 7-item subscales. However, it has not been developed specifically for cancer related fatigue [28]. Mendoza et al., devised Brief Fatigue Inventory which is 9-items numeric scale and is validated for use in mixed cancer population. It measures severity and impact of fatigue during the previous 24 hours [29]. Cancer-Related Fatigue Distress Scale devised by Holley is validated in cancer survivors in which 20 items are measured on a Likert scale of 0-10 that measures physical, social, psychological, cognitive and spiritual distress caused by cancer related fatigue during the previous week [30]. Wu and McSweeney developed an unnamed scale and has shown to be validated in cancer patients and contains of 16-items using a 5-point scale [31].

The multidimensional tools include, Lee Fatigue Scale which is an 18 -item scale assessed on visual analogue scale with 2 subscales fatigue (13-items) and energy (5-items), however not specifically designed for cancer patients [32]. Multidimensional Fatigue Inventory invented by Smets et al., consisting of 20 items where each item is answered on a 7-point Likert score. It characterizes fatigue in terms of general, mental and physical dimensions with internal consistency $(\alpha>0.65)$ and is validated in cancer patients [33]. Functional Assessment of Cancer Therapy-Fatigue assesses both fatigue and its consequences and is a 41-item scale on a 5-point scale of 0-4 which mainly focuses on symptom presence/absence, intensity, affective aspects and perceived interference with functioning ( $\alpha=0.93-0.95)$ [34]. Multidimensional Fatigue Symptom Inventory is validated in cancer patients and allows multiple measurements within a short timescale. It contains 83-items which is assessed on a 5-point scale of 0-4 which characterizes fatigue in terms of global, somatic, cognitive, affective and behavioural aspects in the past week $(\alpha=0.85-0.96)[35,36]$. Fatigue Assessment Questionnaire is another questionnaire which has 20 items, each item assesses on a 4-point scale and characterizes fatigue in terms of physical, affective, and cognitive aspects and is validated in patients with cancer [37]. Revised Piper Fatigue Scale consisting of 22 items assess on an 11-point scale that characterizes fatigue in terms of behavioural/severity, affective meaning, sensory, and cognitive/mood dimensions for entire scale $(\alpha=0.97)$ and subscales well validated in patients with cancer $(\alpha=0.92-0.96)$ [38]. Revised Schwartz Cancer Fatigue Scale designed by Schwartz and Meek consists of 6-items assesses on 1-5 point scale which characterizes fatigue on two dimensions (physical and perceptual) in the previous 2-3 days $(\alpha=0.90)$ and is specifically used in cancer patients [39]. Cancer Fatigue Scale is simple and easy to complete and may be used only with patients currently experiencing fatigue which consists of 15-items, assesses on 1-5 point scale that characterizes fatigue in terms of physical, affective and cognitive dimensions ( $\alpha=$ $0.79-0.89$ ) [40]. Another scale called Fatigue Symptom Inventory is a 13-item scale that assesses on a $0-10$ point scale and evaluates the intensity, duration of fatigue and its impact on quality of life ( $\alpha=$ 0.93-0.95) [41].

The comprehensive and focused assessment primarily includes focused fatigue history that includes onset, pattern, duration, and change over time and associated and alleviating factors. Secondly, evaluating risk of recurrence is based on stage, pathologic factors and treatment history and performing a review of systems to determine if other symptoms substantiate suspicion for recurrence. Thirdly, assessing treatable contributing factors include comorbidities, medications (consider persistent use of sleep aids, pain medications or antiemetics), alcohol, nutritional issues, decreased functional status and deconditioning and lastly, a detailed laboratory evaluation which includes complete blood count, comprehensive metabolic panel and an endocrinologic evaluation.

\section{Role of physiotherapy in cancer related fatigue}

Aerobic exercises: Aerobic exercises are defined as "the rhythmical contraction and relaxation of large muscle groups over a prolonged time for rehabilitation of cancer patients affected by the problem of energy loss". Aerobic exercises have shown to be beneficial for cancer patients who are undergoing treatment. Mock and colleagues reported that home-based walking at a moderate intensity (50-70\% of maximum heart rate), performed for 10 to 45 minutes per day, 4 to 6 days per week, for one to six months, during chemotherapy and radiation treatment for breast cancer reduced cancer related fatigue, sleep disruption, depression, and anxiety while improving 
cardiopulmonary function and QOL. An aerobic training program may break this circle of lack of exercise, impaired performance and easy fatigability. Moreover, physical activity can produce secondary benefits such as improved emotional stability. The aerobic training also increases patients self-confidence and independence. Therefore, cancer patients suffering from primary fatigue should not be advised to increase the amount of daily rest. Rather, they should be counselled to carry out aerobic exercise. Counselling should include a precise definition of duration, intensity and frequency of training [42].

Yoga: Yoga is a promising alternative treatment for cancer survivors with persistent fatigue. Yoga involves physical postures (asanas) that develop strength, flexibility, and relaxation and also promotes present awareness through the focus on body and breath in each pose. Yoga interventions have also demonstrated positive effects on behavioural symptoms related to fatigue, including depressed mood, pain, and physical function. Julienne E. Bower evaluated the acceptability of an Iyengar yoga intervention for fatigued breast cancer survivors which have shown to be well-suited in cancer patients [43].

Pilates: Pilates form of exercises has demonstrated to strengthen the core muscles, subsequently leading to improvement in spine flexibility and posture (Kloubec, 2010). Mind-body exercise can help to improve muscle tone, flexibility, increased circulation and oxygen consumption for more energy, improve posture and stabilize joints to help move with greater ease and confidence. Pilates exercises restore mind and body integration since it is a low impact exercise regimen that strengthens the scapular, abdominal /back and pelvic core musculature while emphasizing deep breathing. Pilates principle emphasises on the idea that if the core is strong, there is an improved ability to use more distal musculature. Other benefits from pilates include lymphatic drainage improvement by its emphasis upon deep breathing along with abdominal exercises which help pump excess lymphatic fluid back towards the heart. It has also demonstrated to strengthen middle back musculature such as the rhomboids and middle trapezius through exercises such as body extension, swan, and diamond postures. This promotes better posture often impaired after breast surgery. Individualized sessions may help in cases of fatigue related to anemia or infection. In addition, pilates has shown accommodate special needs and considerations since it can be performed standing, seated, prone, side-lying, or supine. The supine position is excellent as it provides proprioceptive input to the scapula stabilizers. Proprioception is often decreased after injury or surgery as in cancer patients. Pilates can improve scapulahumeral rhythm including the middle trapezius, rhomboids, and latissimus dorsi. Upper trapezius muscles are often overworked especially post- surgery when pain is a factor. Osteoporosis is a major concern in cancer patients. Many pilates exercises can be modified, adapted, or eliminated from the traditional exercises to improve the same. The emphasis in Pilates is minimal repetitions of an exercise done with correct form. Pilates exercises has shown to be good for cancer patients who are at risk of lymphedema which is the build-up of protein rich fluid in the chest, arm, or armpit after lymph node removal and or radiation or whose fatigue is limiting endurance [44].

Tai chi: Tai Chi Chuan exercises have shown to improve muscular function, cardiopulmonary function, body composition, QOL, bone metabolism, and immune function among breast cancer survivors. A non-controlled, feasibility study showed a positive effect of 10 weeks of Tai Chi Chuan (2 times/week, 60 minutes/session) on memory, attention, and executive function in cancer survivors, suggesting that exercise may be a promising intervention for cognitive function in cancer population [45]. However, more randomized clinical trials are recommended to demonstrate similar results.

Resistance exercises: Resistance exercise involves muscle contraction against resistance leading to improvement in muscular function and bone density. Resistance can be achieved by using dumbbells, resistance bands, or even body weight [46]. Low resistance training is proved to be safe, decreasing side effects, and improves QOL when performed during and following cancer treatment. Studies have shown that after chemotherapy in breast cancer survivors, resistance training, consisting of 2 sets of 8 to 12 repetitions three times per week have resulted in an increase in upper and lower body strength and lean body mass. Courneya and colleagues have demonstrated that breast cancer patients undergoing chemotherapy were able to tolerate higher relative doses of chemotherapy if engaged in resistance exercises [47]. Resistance training has shown to be safe and efficacious for breast cancer survivors who recently completed primary treatment. Schmitz and colleagues implemented a twice weekly resistance training intervention lasting for 6 to 12 months which was found to be safe and resulted in decreased body fat and increased lean body mass [48]. Ahmed and colleagues also assessed the safety of resistance training in breast cancer survivors who had recently completed treatment. Twice weekly resistance training, lasting for 6 months, has shown no change in arm circumference in participants and therefore did not seem to contribute to the development of lymphedema [49]. Breast cancer survivors who performed progressive moderate intensity resistance training in addition to jump/impact training three times per week for one year benefited from a reduction in fracture risk due to preservation of bone mineral density at the lumbar spine. This preservation may be particularly beneficial for breast cancer survivors taking aromatase inhibitors [50]. Resistance training performed 3 days per week for 12 weeks by prostate cancer survivors receiving androgen deprivation therapy have shown to improve cancer related fatigue, cognitive function, QOL, and muscular strength [51].

Acupuncture: Acupuncture has an effect on inflammatory cytokines, T- lymphocytes, and various peptides. A recent study suggested that cytokines and tumour necrosis factor $\alpha$ are contributing factors in the development of fatigue. Study by Molassiotis A et al., determined that women with breast cancer and cancer related fatigue reported significant improvements in overall fatigue, physical and mental fatigue, activity, motivation, psychological distress, and all domains of quality of life after 6 weeks of acupuncture. The improvements in psychological distress and emotional adjustment are important, particularly because fatigue has shown to be part of symptom clusters along with anxiety and depression, and acupuncture could be an approach to manage clusters of symptoms rather than only single symptom. It is also important to emphasize that acupuncture is not just a simple needling technique eliciting specific physiologic effects, but wide improvements in patient-reported outcomes may indicate nonspecific placebo effects. Acupuncture could be a treatment option for cancer related fatigue, although its availability 
may be an issue in many health care services and patients may not be able to afford private costs. Future research should concentrate on cost-effectiveness of acupuncture, because potentially expensive acupuncture treatments may be offset by reduced societal costs and health care utilization in those suffering significant and protracted fatigue after treatment [52].

\section{Conclusion}

Physical activity in the form of aerobic exercises, yoga, pilates, taichi, resistance exercises and acupuncture has proved to be effective in cancer survivors experiencing cancer related fatigue, psychological factors and also in improving quality of life. More clinical trials with larger sample size in different cancer populations may help to document more evidence in relation to importance of physiotherapy during and after cancer treatment.

\section{References}

1. Smets E, Garssen B, Schuster-Uitterhoeve A, de Haes J (1993) Fatigue in cancer patients. Br J Cancer 68: 220-224.

2. Holmes MJ, Diack DS, Easingwood RA, Cross JP, Carlisle B (1997) Electron microscopic immunocytological profiles in chronic fatigue syndrome. $J$ Psychiatr Res 31: 115-122.

3. Jacobsen PB1, Hann DM, Azzarello LM, Horton J, Balducci L, et al. (1999) Fatigue in woman receiving adjuvant chemotherapy for breast cancer: characteristics, course and correlates. J Pain Symptom Manage 18: 233242.

4. Stone P, Hardy J, Huddart R, A'Hern R, Richards M (2000) Fatigue in patients with prostate cancer receiving hormone therapy. Eur J Cancer 36: 11341141.

5. Sitzia J, Huggins L (1998) Side effects of cyclophosphamide, methotrexate 5-fluorouracil (CMF) chemotherapy for breast cancer. Cancer Pract 6: 13-21.

6. Robinson KD, Posner JD (1992) Patterns of self-care needs and interventions related to biologic response modifier therapy: fatigue as a model. Semin Oncol Nurs 8: 17-22.

7. Stone P1, Hardy J, Broadley K, Tookman AJ, Kurowska A, et al. (1999) Fatigue in advanced cancer: a prospective controlled cross-sectional study. Br J Cancer 79: 1479-1486.

8. Broeckel JA, Jacobsen PB, Horton J, Balducci L, Lyman GH (1998) Characeristics and correlates of fatigue after adjuvant chemotherapy for breast cancer. J Clin Oncol 16: 1689-1696.

9. Mock V, Atkinson A, Barsevick A, Cella D, Cimprich B, et al. (2000) NCCN practice guidelines for cancer-related fatigue. Oncology 14: 151-161.

10. National Comprehensive Cancer Network (2003) National Comprehensive Cancer Network practice guidelines cancer-related fatigue panel guidelines. Rockledge: National Comprehensive Cancer Network.

11. Cella D, Peterman A, Passik S, Jacobsen P, Breitbart W (1998) Progress towards guidelines for the management of fatigue. Oncology 12: 369-377.

12. Portenoy RK, Itri L (1999) Cancer-related fatigue: guidelines for evaluation and management. Oncologist 4: 1-10.

13. Ahlberg K, Ekman T, Gaston-Johansson F, Mock V (2003) Assessment and management of cancer-related fatigue in adults. The Lancet 362: 640-650.

14. Dimeo FC (2001) Effects of Exercise on Cancer-Related Fatigue. Cancer Supplement 92: 1689-1693.

15. Mock V, Pickett M, Ropka ME, Muscari Lin E, Stewart KJ, et al. (2001) Fatigue and quality of life outcomes of exercise during cancer treatment. Cancer Pract 9: 119-127

16. Berger AM, Farr L (1999) The influence of daytime inactivity and night time restlessness on cancer-related fatigue. Oncol Nurs Forum 26: 1663-1671.
17. Bower JE, Ganz PA, Desmond KA, Rowland JH, Meyerowitz BE, et al. (2000) Fatigue in breast cancer survivors: Occurrence, correlates, and impact on quality of life. J Clin Oncol 18: 743-753.

18. Servaes P, Verhagen S, Bleijenberg G (2002) Determinants of chronic fatigue in disease-free breast cancer patients: A cross-sectional study. Ann Oncol 13: $589-598$

19. Mendoza TR, Wang XS, Cleeland CS, Morrissey M, Johnson BA, et al. (1999) The rapid assessment of fatigue severity in cancer patients: Use of the Brief Fatigue Inventory. Cancer 85: 1186-1196.

20. Nekolaichuk C, Watanabe S, Beaumont C (2008) The Edmonton Symptom Assessment System: A 15-year retrospective review of validation studies. Palliat Med 22: 111-122.

21. McCorkle R, Quint-Benoliel J (1983) Symptom distress, current concerns and mood disturbance after diagnosis of life-threatening disease. Soc Sci Med 17: 431-438.

22. de Haes JC, van Knippenborg FC, Neijt JP (1990) Measuring psychological and physical distress in cancer patients: Structure and application of the Rotterdam Symptom Checklist. Br J Cancer 62: 1034-1038.

23. Aaronson NK, Ahmedzai S, Bergman B, Bullinger M, Cull A, et al. (1993) The European Organization for Research and Treatment of Cancer QLQ-C30: A quality-of-life instrument for use in international clinical trials in oncology. J Natl Cancer Inst 85: 365-376.

24. McHorney CA, Ware JE Jr, Raczek AE (1993) The MOS 36-Item ShortForm Health Survey (SF-36): II. Psychometric and clinical tests of validity in measuring physical and mental health constructs. Med Care 31: 247-263.

25. Cleeland CS, Mendoza TR, Wang XS, Chou C, Harle MT, et al. (2000) Assessing symptom distress in cancer patients: The M.D. Anderson Symptom Inventory. Cancer 89: 1634-1646.

26. Kirsh KL, Passik S, Holtsclaw E, Donaghy K, Theobald D (2001) I get tired for no reason: A single item screening for cancer-related fatigue. J Pain Symptom Manage 22: 931-937.

27. Rhoten D (1982) Fatigue and the postsurgical patient. In: Norris CM, ed. Concept Clarification in Nursing. Rockville, MD: Aspen Systems Corporation 277-300.

28. McNair DM, Lorr M, Droppleman L (1992) Profile of Mood States, Second Edition. San Diego, CA: Educational and Industrial Testing Service 1-40.

29. Mendoza TR, Wang XS, Cleeland CS, Morrissey M, Johnson BA, et al. (1999) The rapid assessment of fatigue severity in cancer patients: Use of the Brief Fatigue Inventory. Cancer 85: 1186-1196.

30. Holley SK (2000) Evaluating patient distress from cancer-related fatigue: An instrument development study. Oncol Nurs Forum 27: 1425-1431.

31. Wu HS, McSweeney M (2004) Assessing fatigue in persons with cancer: An instrument development and testing study. Cancer 101: 1685-1695.

32. Lee KA, Hicks G, Nino-Murcia G (1991) Validity and reliability of a scale to assess fatigue. Psychiatry Res 36: 291-298.

33. Smets EM, Garssen B, Bonke B, De Haes JC (1995) The Multidimensional Fatigue Inventory (MFI): Psychometric qualities of an instrument to assess fatigue. J Psychosom Res 39: 315-325.

34. Yellen SB, Cella DF, Webster K, Blendowski C, Kaplan E (1997) Measuring fatigue and other anaemia related symptoms with the Functional Assessment of Cancer Therapy (FACT) measurement system. J Pain Symptom Manage 13: $63-74$.

35. Stein KD, Martin SC, Hann DM, Jacobsen PB (1998) A multidimensional measure of fatigue for use with cancer patients. Cancer Pract 6: 143-152.

36. Stein KD, Jacobsen PB, Blanchard CM, Thors C (2004) Further validation of the multidimensional fatigue symptoms inventory-short form. J Pain Symptom Manage 27: 14-23.

37. Glaus A (1998) Fatigue in patients with cancer. Analysis and assessment. Recent Results Cancer Res 145: 1-172. 
38. Piper BF, Dibble SL, Dodd MJ, Weiss MC, Slaughter RE (1998) The revised Piper Fatigue Scale: Psychometric evaluation in women with breast cancer. Oncol Nurs Forum 25: 677-684

39. Schwartz A, Meek P (1999) Additional construct validity of the Schwartz Cancer Fatigue Scale. J Nurs Meas 7: 35-45.

40. Okuyama T, Akechi T, Kugaya A, Okamura H, Shima Y, et al. (2000) Development and validation of the Cancer Fatigue Scale: A brief, threedimensional, self-rating scale for assessment of fatigue in cancer patients. J Pain Symptom Manage 19: 5-14.

41. Hann DM, Jacobsen PB, Azzarello LM, Martin SC, Curran SL, et al. (1998) Measurement of fatigue in cancer patients: Development and validation of the Fatigue Symptom Inventory. Qual Life Res 7: 301-310.

42. Hann DM, Denniston MM, Baker F (2000) Measurement of fatigue in cancer patients: Further validation of the Fatigue Symptom Inventory. Qual Life Res 9: 847-854.

43. Bower JE, Garet D, Sternlieb B (2011) Yoga for Persistent Fatigue in Breast Cancer Survivors: Results of a Pilot Study. Evid Based Complement Alternat Med 623168

44. Stan DL, Rausch SM, Sundt K, Cheville AL, Youdas JW, et al. (2012) Pilates for Breast Cancer Survivors: Impact on Physical Parameters and Quality of Life after Mastectomy. Clin J Oncol Nurs 16: 131-141.

45. Mustian KM, Sprod LK, Janelsins M, Peppone LJ, Mohile S (2013) Exercise Recommendations for Cancer-Related Fatigue, Cognitive Impairment, Sleep problems, Depression, Pain, Anxiety, and Physical Dysfunction: A Review. Oncol Hematol Rev 8: 81-88.
46. Thompson WR, Gordon N (2010) ACSM's guidelines for exercise testing and prescription. $8^{\text {th }}$ ed. Lippincott Williams \& Wilkins; Philadelphia

47. Courneya KS, Segal RJ, Gelmon K, Reid RD, Mackey JR, et al. (2007) Sixmonth follow-up of patient-rated outcomes in a randomized controlled tria of exercise training during breast cancer chemotherapy. Cancer Epidemiol Biomarkers Prev 16: 2572-2578.

48. Schmitz KH, Ahmed RL, Hannan PJ, Yee D (2005) Safety and efficacy of weight training in recent breast cancer survivors to alter body composition, insulin, and insulin-like growth factor axis proteins. Cancer Epidemiol Biomarkers Prev 14: 1672-1680.

49. Ahmed RL, Thomas W, Yee D, Schmitz KH (2006) Randomized controlled trial of weight training and lymphedema in breast cancer survivors. J Clin Oncol 24: 2765-2772.

50. Winters-Stone KM, Dobek J, Nail L, Bennett JA, Leo MC, et al. (2011) Strength training stops bone loss and builds muscle in postmenopausal breast cancer survivors: a randomized, controlled trial. Breast Cancer Res Treat 127: 447-456.

51. Segal RJ, Reid RD, Courneya KS, Malone SC, Parliament MB, et al. (2003) Resistance exercise in men receiving androgen deprivation therapy for prostate cancer. J Clin Oncol 21: 1653-1659.

52. Molassiotis A, Bardy J, Finnegan-John J, Mackereth $P$, Ryder DW, et al. (2012) Acupuncture for Cancer-Related Fatigue in Patients With Breast Cancer: A Pragmatic Randomized Controlled Trial. J Clin Oncol 30: 4470 4476.

Copyright: (c) 2016 Pattanshetty RB, at al. This is an open-access article distributed under the terms of the Creative Commons Attribution License, which permits unrestricted use, distribution, and reproduction in any medium, provided the original author and source are credited. 